\title{
Degradation of naturally occurring and engineered antimicrobial peptides by proteases
}

\author{
Bernard J. Moncla ${ }^{1,2 *}$, Kara Pryke ${ }^{2}$, Lisa Cencia Rohan ${ }^{1,2,3}$, Phillip W. Graebing ${ }^{2}$ \\ ${ }^{1}$ Department of Obstetrics, Gynecology and Reproductive Sciences, University of Pittsburgh, Pittsburgh, USA; \\ ${ }^{2}$ Magee-Womens Research Institute, Pittsburgh, USA; \\ ${ }^{3}$ Department of Pharmaceutical Sciences, University of Pittsburgh, Pittsburgh, USA. \\ Email: “bjm4@pitt.edu, kpryke@mwri.magee.edu, lrohan@mwri.magee.edu, pgraebing@mwri.magee.edu
}

Received 3 September 2011; revised 19 October 2011; accepted 8 November 2011.

\begin{abstract}
We hypothesized that current antimicrobial peptides should be susceptible to proteolytic digestion. The antimicrobial peptides: Griffithinsin, RC-101, LL-37, LSA-5, PSC-RANTES and DJ007 were degraded by commercially available proteases. Two different species of anaerobic vaginal flora, Prevotella bivia and Porphyromonas asaccharolytica also degraded the materials. Griffithsin was resistant to digestion by 8 of the 9 proteases and the bacteria while LL-37 was the most sensitive to protease digestion. These data suggests most of the molecules may not survive for very long in the proteolytic rich environments in which they are intended to function.
\end{abstract}

Keywords: Microbicides; HIV; Anti-HIV; Antimicrobial Peptides; Proteases

\section{INTRODUCTION}

One of the approaches to curbing the spread of HIV is the development of intra-vaginal microbicides. When available these drugs will allow women to use them without the knowledge of their partners and will help prevent coerced unsafe sex. Early microbicide studies focused on peptides such as: LL-37, LSA-5, RC-101, PSC-RANTES and Griffithsin. LL-37 a cathelicidin, and LSA-5, a cathelicidin derivative, both have a broad spectrum of activity killing both bacteria and viruses. Other anti-HIV microbicides such as RC-101 and PSC-RANTES prevent viral entry into the cell, were derived from mammalian tissues. Griffithsin, a lectin isolated from the red algae Griffithsia, is another entry level inhibitor.

Clinical and preclinical trials are expensive and time consuming. It is therefore important to screen potential molecules to ensure they will persist and remain active in vivo. Many groups are developing peptide based micro- bicides using naturally occurring and engineered antimicrobial peptides (AMPs). Most of these efforts have been directed towards increasing potency and in some cases protecting the molecules from degradation [1-4]. Many AMPs have potent anti-HIV activity in vitro but require much higher concentrations in vivo.

Peptidases are ubiquitous in the human body and are involved in simple functions such as food digestion and in regulation of complex processes such as apoptosis and the complement cascade [5]. They function to turn processes on and off. Proteases also originate from the microflora in the mouth, vagina and GI tract, all sites of potential HIV infection. Protease activity in the normal vagina has been reported to be $0.16 \mathrm{U}$ and increases to 0.29 in women with bacterial vaginosis [6]. Infection by trichomonads, Candida and Neisseria gonorrhea lead to higher vaginal protease levels [7]. Endogenous proteases such as the Kalledreins have been measured as high as $7.7 \mathrm{mg} / \mathrm{l}$ of vaginal fluid [8].

Although peptidases are many, their mechanisms of action are few; therefore we selected peptidases to represent the different enzyme classes and determined their effectiveness in degrading different AMPs. We also used four representative vaginal bacteria to determine whether or not they would alter the two most protease resistant AMPs. There have been very few studies to reporting the actual stability of AMP to proteolytic attack. In these studies we report a simple method for the screening of AMPs for resistance to proteases.

\section{MATERIALS AND METHODS}

\subsection{Enzymes and Chemicals}

Enzymes and chemicals were all obtained from SigmaAldrich, (Sigma-Aldrich, St. Louis, MO 63178, USA). AMPs RC-101, LL-37, LSA-5, and PSC-RANTES were synthesized at the University of Pittsburgh Peptide Synthesis Core Facility (Pittsburgh, PA), Griffithsin was kindly provided by Kenneth Palmer, James Graham Brown 
Cancer Center, University of Louisville (Louisville, KY.) and DJ007 was provided by Jonathon Holt of The International Partnership for Microbicides (Silver Spring, MD 20910, USA).

\subsection{Protease Digestions SDS-PAGE}

Proteinases were suspended in the appropriate buffer at a concentration of $1 \mathrm{mg} / \mathrm{ml}$ or used as supplied by the manufacturer and held on ice. The enzymes used were: Trypsin, Papain, Endoproteinase Lys-C, Leucine aminopepdidase, Elastase (human leukocyte), Proteinase K, $\alpha$-Chymotrypsin and Pronase (Streptococcus grieus). Enzyme solutions were diluted to $1 \mu \mathrm{U}$ immediately prior to use; level of protease that could be encountered in vivo. AMPs, $5 \mu \mathrm{g}$, were diluted with buffer to $15 \mu \mathrm{l}$ and $15 \mu$ l enzyme solution added and mixed on a reciprocating shaker for $1 \mathrm{hr}$. at $37^{\circ} \mathrm{C}$. Reactions were stopped by the addition of $60 \mu$ l of Tris Tricine sample buffer (Bio-Rad, Hercules, CA) and placing in a boiling water bath for $5 \mathrm{~min}$. Controls samples received buffer without enzyme but were otherwise treated the same.

Samples were evaluated by SDS-PAGE using BioRad Tris Tricine gradient gels (10\% to $20 \%$ acrylamide). Peptide degradation was considered positive if the peptide bands disappeared from the gel after proteinase treatment. Preliminary studies demonstrated that not all the AMPs stained with our silver stain therefore Coomassie Brilliant Blue-R was used for staining.

\subsection{Digestion with Bacteria}

Prevotella bivia strains ATCC 29303 and HSP-1174-1b and Porphyromonas asaccharolytica strains 11-B-E and CRIS8A-3A were cultured anaerobically overnight on Brucella agar (PML Laboratories, Hercules, CA USA) and used to prepare suspensions at a density of $0.5 \mathrm{Mc}-$ Farland units (approx. $10^{8} / \mathrm{ml}$ ) in ACES buffer, pH 7.2. AMPs were prepared as described above and $15 \mu$ added to $15 \mu \mathrm{l}$ of bacterial suspension, mixed and then incubated with shaking at $37^{\circ} \mathrm{C}$ for $1 \mathrm{hr}$. Cells were separated by centrifugation through a Microcon centrifugal filter (Millipore, Bedford MA USA) with a 30,000 molecular mass cut off. Controls for the binding of drug to the bacteria were prepared as described but immediately separated by the filtration. The samples were then analyzed by SDS-PAGE as described above.

\section{RESULTS}

In most cases the results were easily interpreted, the exception was the digestion of PSC-RANTES by trypsin. In these experiments the PSC-RANTES bands appeared to have a reduced intensity or density rather than disappearing (see Figure 1). Figure 1 presents the results from a typical experiment. The results of all enzyme ex- periments are presented in Table 1. In Table 1 the drugs have been listed with those sensitive to proteolytic digestion to the left and the most resistant on the right. Serine proteases are the most common proteases found in humans and overall the materials tested were sensitive to degradation by serine proteases while GRFT was resistant to all the serine proteases and degraded by 1 of 2 metalloproteases examined. Of the remaining drugs, all were degraded by all of the serine proteases except LSA-5 which was not digested by endopeptidase C and RC-101 was not degraded by chymotrypsin. LSA-5, LL37 and PSC-RANTES were degraded by members of all of the classes of proteases tested. Although RC-101 was degraded by all of the serine proteases it was not degraded by any of the other classes of enzyme tested. GRFT was only degraded by elastase while LL-37 was degraded by all enzymes tested. PSC-RANTES was degraded by all enzymes except Leu. aminopeptididase. LSA- 5 was resistant to 2 of the enzymes tested and the RC-101 and DJ007 were degraded by one half of the proteases Results, presented in Figure 2 clearly demonstrate the degradation of PSC-RANTES but not GRFT and that the drugs were not bound to the bacteria. RC-101 was also degraded (data not shown).

\section{DISCUSSION}

The enzymes used in this study were selected to represent the different classes and specific types found in the human body. To determine what contribution vaginal flora might play in degradation of these molecules we used GRFT, PSC-RANTES and RC-101 and exposed them to four representative strains of vaginal bacteria. These results were not surprising since a great number of vaginal anaerobes produce many different proteases [9]. Considering that anaerobic plate counts in cervical vaginal lavages and vaginal fluid may reach $10^{8}$ to $10^{9}$ per ml there is certainly reason to be concerned that the AMPs may not persist for any considerable length of time. Thus, the great number of different sources for proteases in vaginal fluids presents a daunting task of preventing proteolytic attack [7-10]. It is tempting to postulate that the large quantities of protein in vaginal fluid would prevent proteolysis of the drugs by overwhelming any proteases; however, most human proteins are glycosylated which prevents proteolysis [11]. Our attempts to investigate this possibility were unfruitful when we combined cervical mucus, AMP and protease. These experiments were difficult to interpret. Although the AMPs appear to decrease in quantity in the gels, so did many of the mucus protein. We are unwilling to make any conclusions without further extensive experimentation. The resistance of the Griffithsin to proteolytic attack may be a reflection of different evolutionary paths 


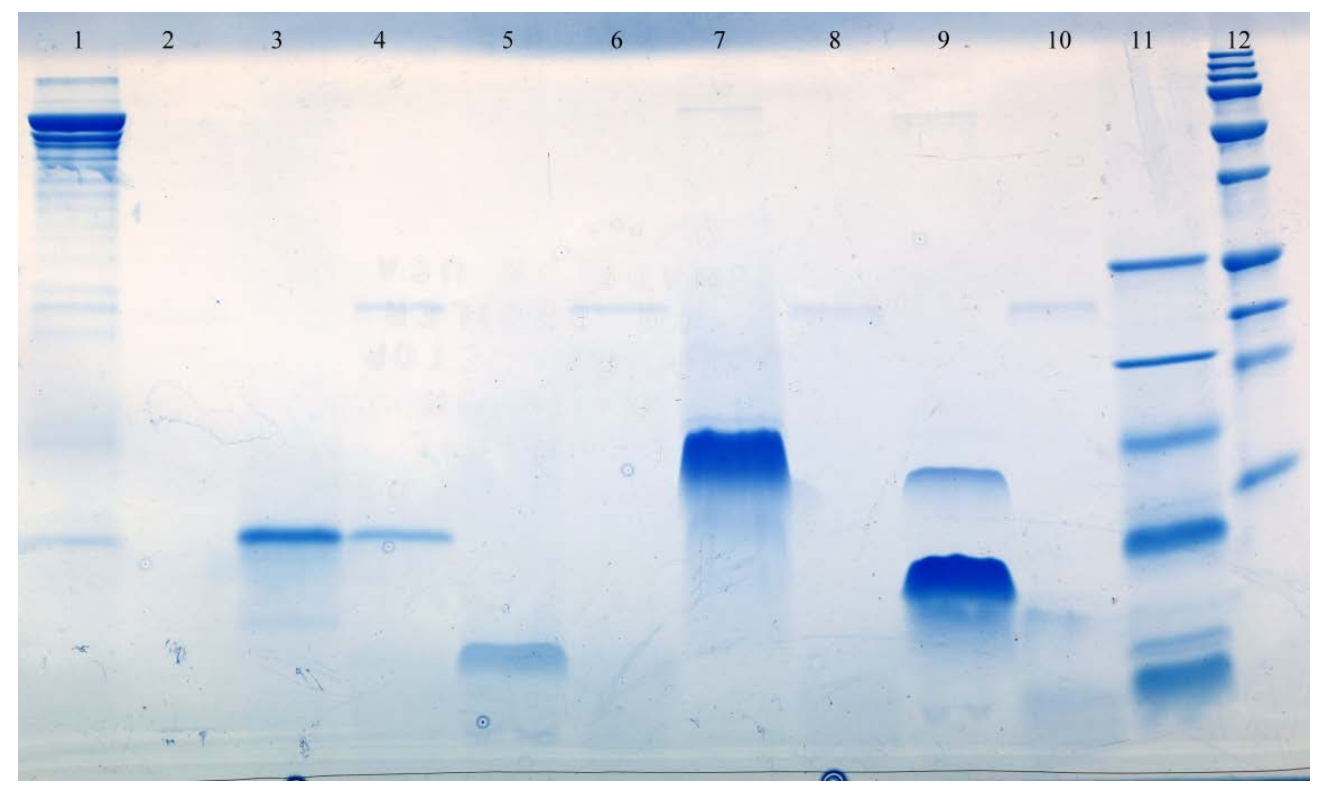

Figure 1. SDS-PAGE analysis of antimicrobial peptides by trypsin. Lane 1, BSA; Lane 2, BSA plus typsin; Lane 3, PSC-RANTES; Lane 4, PSC-RANTES trypsin treated; Lane 5, RC101; Lane 6, trypsin treated RC101; Lane 7, LSA-5; Lane 8, trypsin treated LSA-5; 9, LL-37; Lane 10, trypsin treated; Lane 11, low molecular mass standard; Lane 12, high molecular mass standards. The molecular weights of these standards were: 250,000, 150,000, 100,000, 75,000, 37,000, 25,000, 20,000, 15,000 and 10,000. The molecular weights of the low standards (lane 11) were 26,626, 16,950, 14,437, 6512, 3496 and 1423.

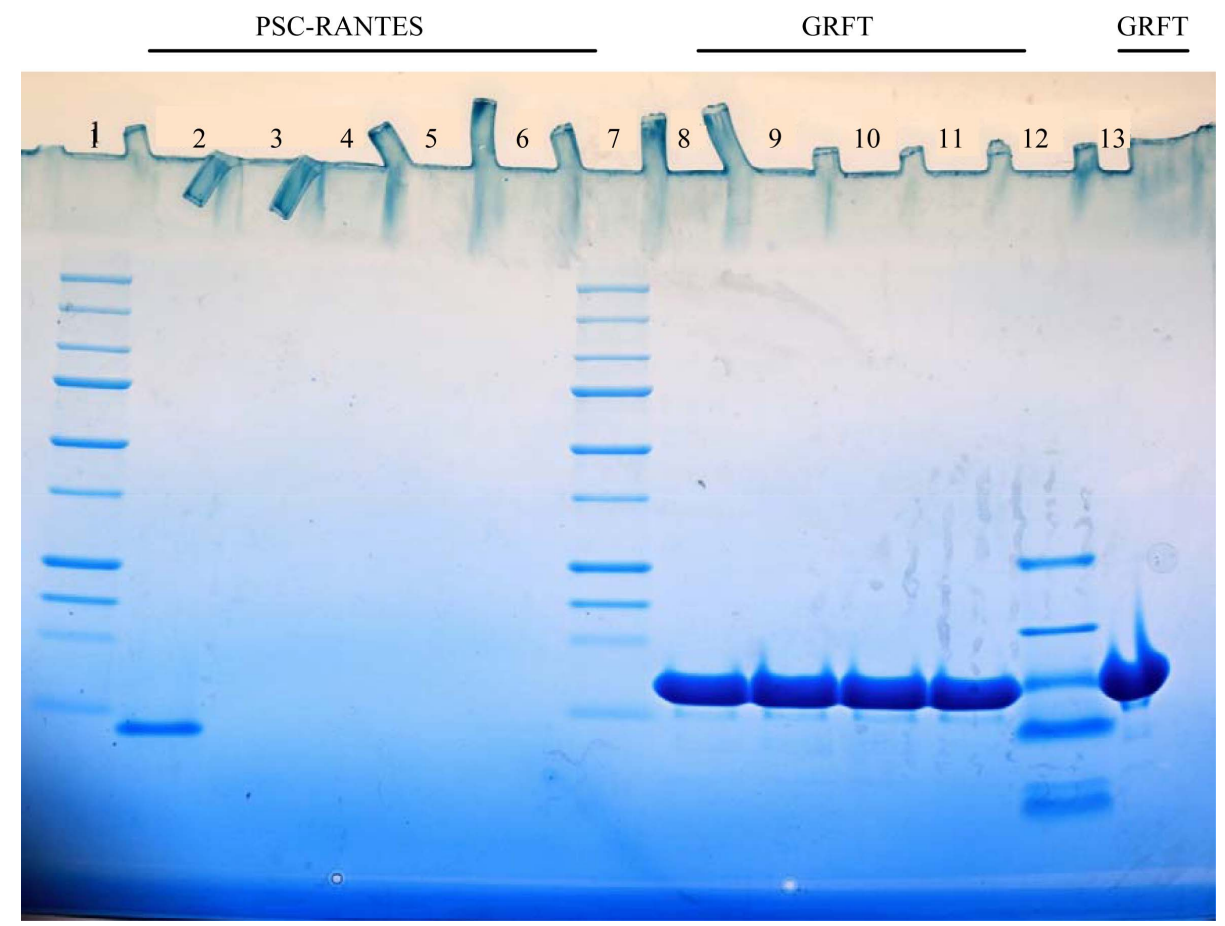

Figure 2. Hydrolysis of PSC-RANTES and GRFT by strains of Prevotella bivia and Porphyromonas asaccharolytica. Lanes 1 and 7 high molecular mass protein standards. Lane 12, low molecular mass standard. Lanes 2 and 8, PSC-RANTES and GRTF controls (see text). Lane 3 through 6 bacteria plus PSC-RANTES and lanes 8 to 11 GRTF plus bacteria. Bacteria suspensions were incubated with $15 \mu \mathrm{l}$ of indicated material as described in the texted. Bacteria used were: P. bivia HSP-1174-1b; lanes 3 and 9; P. bivia 29303 lanes 6 and 13, lanes; P. asaccharolytica CRIS 8A-3-E, lanes 5 and 11; P. asaccharolytica. 11-bE lanes 4 and 9. 
Table 1. Hydrolysis of microbicides by proteases.

\begin{tabular}{|c|c|c|c|c|c|c|c|}
\hline \multirow[b]{2}{*}{ Protease used } & \multicolumn{7}{|c|}{ Materials tested } \\
\hline & Protease family ${ }^{1}$ & $11-37$ & PSC-RANTES & DJ007 & LSA-5 & RC101 & GRFT \\
\hline Pepsin & A & $+^{3}$ & + & NA & + & - & - \\
\hline Papain & $\mathrm{C}$ & + & + & - & + & - & - \\
\hline Elastase & M & + & + & NA & + & - & + \\
\hline Leucine aminopepdidase & M & + & - & NA & - & - & - \\
\hline Pronase $^{2}$ & S & + & + & + & + & + & - \\
\hline$\alpha$-chymotrypsin & S & + & + & + & + & - & - \\
\hline Proteinase K & S & + & + & + & + & + & - \\
\hline Endoproteinase & S & + & + & + & - & + & - \\
\hline \multicolumn{8}{|l|}{ Lys-C } \\
\hline Trypsin & S & + & $+/-$ & NA & + & + & - \\
\hline Number of proteases active against AMPs & & $9 / 9$ & $8 / 9$ & 4 of 5 & $7 / 9$ & $4 / 9$ & $1 / 9$ \\
\hline Number active/No. tested & & & & & & & \\
\hline
\end{tabular}

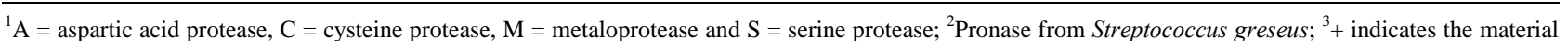
was hydrolyzed by the indicated protease, “-” indicates no hydrolysis. NA indicates the enzyme was not active in $2 \%$ SDS containing buffer that is required to solubilize the material.

of mammals and algae.

The extension of these observations to other potential sites of infection, the oral cavity and the lower bowel may or may not be warranted. The levels of protease in saliva are detectable but probably too low to lead to proteolytic attack. The periodontal disease associated bacteria Tannerella forsythia and Porphyromonas gingivalis both produce a trypsin-like protease [13,14]; however, the enzyme is probably confined to the periodontium. The lower bowel has very high bacterial numbers and can be expected to provide a hostile environment for peptides.

\section{CONCLUSIONS}

The great abundance and variety of protease types at sites intended for microbicide use is well documented. These preliminary results suggest that peptide based microbicides should be thoroughly investigated regarding their susceptibility to degradation by proteases. The GRFT was the most promising material tested; however, degradation by human derived Leukocyte elastase is cause for concern. As a group there may not be much promise for further development of such compounds unless they can be formulated in a way that protects them from proteolytic attack.

\section{ACKNOWLEDGEMENTS}

Parts of this work were presented at the Microbicides 2010 Conference in Pittsburgh, PA. USA.
This work was supported by grants from The International Partnership for Microbicides, CONRAD, Pendleton Charitable Trust Fund, NIH grants: AI068633, AI39061 and AI076169.

\section{REFERENCES}

[1] Deslouches, B., Phadke, S.M., Lazarevic, V., Cascio, M., Islam, K., Montelaro, R.C., et al. (2005) De novo generation of cationic antimicrobial peptides: influence of length and tryptophan substitution on antimicrobial activity. Antimicrobial Agents and Chemotherapy, 49, 316322. doi:10.1128/AAC.49.1.316-322.2005

[2] Lim, J.K., Lu, W., Hartley, O. and DeVico, A.L. (2006) $\mathrm{N}$-terminal proteolytic processing by cathepsin $\mathrm{G}$ converts RANTES/CCL5 and related analogs into a truncated 4-68 variant. Journal Leukocyte Bioliology, 80, 13951404. doi:10.1189/jlb.0406290

[3] Oh, J.E., Hong, S.Y. and Lee, K.H. (1999) Structureactivity relationship study: Short antimicrobial peptides. Journal Peptide Ressearch, 53, 41-46. doi:10.1111/j.1399-3011.1999.tb01615.x

[4] Patton, D.L., Cosgrove Sweeney, Y.T., McCarthy, T.D. and Hillier, S.L. (2006) Preclinical safety and efficacy assessments of dendrimer-based (SPL7013) microbicide gel formulations in a nonhuman primate model. Antimicrobial Agents and Chemotherapy, 50, 1696-1700. doi:10.1128/AAC.50.5.1696-1700.2006

[5] Potempa, J. and Pike, R.N. (2009) Corruption of Innate Immunity by Bacterial Proteases. Journal of Innate Immunity, 1, 70-87. doi:10.1159/000181144

[6] Howe, L., Wiggins, R., Soothill, P.W., Millar, M.R., Horner, P.J. and Corfield, A.P. (1999) Mucinase and sialidase activity of the vaginal microflora: Implications for the 
pathogenesis of preterm labour. International Journal of STD and AIDS, 10, 442-447.

doi:10.1258/0956462991914438

[7] Bernardis, F.D., Agatensi, L., Ross, I.K., Emerson, G.W., Lorenzini, R. and Sullivan, P.A., et al. (1990) Evidence for a role for secreted aspartate proteinase of candida albicans in vulvovaginal Candidiasis. The Journal of Infectious Diseases, 161, 1276-1283. doi:10.1093/infdis/161.6.1276

[8] Shaw, J.L.V., Petraki, C., Watson, C., Bocking, A. and Diamandis, E.P. (2008) Role of tissue kallikrein-related peptidases in cervical mucus remodeling and host defense. Biological Chemistry, 389, 1513-1522.

doi:10.1515/BC.2008.171

[9] Steffen, H., Rieg, S., Wiedemann, I., Kalbacher, H., Deeg, M., Sahl, H.G., et al. (2006) Naturally processed dermcidin-derived peptides do not permeabilize bacterial membranes and kill microorganisms irrespective of their charge. Antimicrobial Agents and Chemotherapy, 50, 26082620. doi:10.1128/AAC.00181-06

[10] Blake, M., Holmes, K.K. and Swanson, J. (1979) Studies on gonococcus infection. XVII. Igaâ, -cleaving pro- tease in vaginal washings from women with gonorrhea. The Journal of Infectious Diseases, 139, 89-92. doi:10.1093/infdis/139.1.89

[11] Katz, F.N., Rothman, J.E., Lingappa, V.R., Blobel, G. and Lodish, H.F. (1977). Membrane assembly in vitro: Synthesis, glycosylation, and asymmetric insertion of a transmembrane protein. Proceedings of the National Academy of Sciences of the United States of America, 74, 3278-3282. doi:10.1073/pnas.74.8.3278

[12] Kaufman, E. and Lamster, I.B. (2000) Analysis of saliva for periodontal diagnosis. Journal of Clinical Periodontology, 27, 453-465. doi:10.1034/j.1600-051x.2000.027007453.x

[13] Braham, P.H. and Moncla, B.J. (1992) Rapid presumptive identification and further characterization of Bacteroides forsythus. Journal of clinical microbiology, 30, 649-654.

[14] Moncla, B.J., Braham, P., Rabe, L.K. and Hillier, S.L. (1991) Rapid presumptive identification of black-pigmented gram-negative anaerobic bacteria by using 4methylumbelliferone derivatives. Journal of clinical microbiology, 29, 1955-1958. 